\title{
A new optimization model for market basket analysis with allocation considerations: A genetic algorithm solution approach
}

\author{
Majeed HEYDARI \\ University of Zanjan, Zanjan, Iran \\ mheydari@znu.ac.ir
}

Amir YOUSEFLI

Imam Khomeini International University, Qazvin, Iran

yousefli@soc.ikiu.ac.ir

\begin{abstract}
Nowadays market basket analysis is one of the interested research areas of the data mining that has received more attention by researchers. But, most of the related research focused on the traditional and heuristic algorithms with limited factors that are not the only influential factors of the basket market analysis. In this paper to efficient modeling and analysis of the market basket data, the optimization model is proposed with considering allocation parameter as one of the important and effectual factors of the selling rate. The genetic algorithm approach is applied to solve the formulated non-linear binary programming problem and a numerical example is used to illustrate the presented model. The provided results reveal that the obtained solutions seem to be more realistic and applicable.
\end{abstract}

Keywords: market basket analysis, association rule, non-linear programming, genetic algorithm, optimization.

Please cite the article as follows: Heydary, M. and Yousefli, A. (2017), "A new optimization model for market basket analysis with allocation considerations: A genetic algorithm solution approach", Management \& Marketing. Challenges for the Knowledge Society, Vol. 12, No. 1, pp. 111. DOI: $10.1515 / \mathrm{mmcks}-2017-0001$

\section{Introduction}

Nowadays, data mining is widely used in several aspects of science such as manufacturing, marketing, CRM, retail trade etc. Data mining or knowledge discovery is a process for data analyzing to extract information from large databases. Artificial intelligence, neural network, statistical techniques, pattern recognition, clustering and classification approaches are areas included in the data mining. With increasing data mining popularity, most researchers apply data mining techniques to extract information from data sets.

Focusing on the application of operation research in data mining, some authors used mathematical programming approaches to discover knowledge. Mangasarian (1965) applied optimization models to data classification and Vinod (1964) and Rao (1971) also used some optimization models for clustering. Padmanabhan and Tuzhilin (2003) used optimization model to address eCRM problems. Bradley et al. (1999) also formulated the basic categories of data mining methods as optimization problems. Olafsson et al. (2008) surveyed the intersection of operation research and data mining and they illustrated the range of interactions between them. On the other side, some authors focused on association rule mining as one of the most important techniques of data mining to identify association rules which fulfill the predetermined minimum support and confidence from a given database (Kotsiantis and Kanellopoulos, 2006). Hegland (2003) reviewed the most famous algorithms for producing association rules. 
Market basket analysis as a subset of market research is a well-known problem that numerous researchers have paid special attention to until now. For more information on market research and its trends please refer to Barbu (2013). Reviewing the reported research shows that data mining techniques are widely used to handle the raised problems in market basket analysis. For example, Tang et al. (2008) proposed an approach to performing market basket analysis in a multi-store and multi-period environment. In another attempt, Chen et al. (2005) claimed that the most of the models presented for dealing with market basket problem could not discover important purchasing patterns when multiple stores exist. So they developed a method to overcome this weakness. Yun et al. (2006) clustered data of market basket using a novel measurement that they named category-based adherence. Cavique (2007) converted market basket problem into a maximum-weighted clique problem for discovering large item set patterns. Russell and Urban (2010) presented an optimization model for shelfspace management problem in which products are grouped as families and the location of each family is determined on the shelf. They considered shelf location effect on sales but did not attend the cross-selling effect and also purchase data never been used. Nierop et al. (2008) proposed a method for dealing with shelf- space management problem which consists of two parts. In the first phase, a statistical model was provided to measure the impact of shelf layout on sales. In the second part simulated annealing (SA) is used to maximize expected total profit. Similar to Russell and Urban (2010), they did not mind association rules from customers' purchasing data to maximize the crossselling effect.

In the most recent research, considering a market basket analysis problem, Saraf and Patil (2016) proposed a bottom-up hierarchical clustering approach for clustering retail items. To do this, they applied the concept of 'distance' between the entities or, groups of entities to achieve the purpose of market-basket analysis. Market basket analysis is not only the subject of some research, but also the concept of market basket analysis was used by some researcher to other applicable problems. For example, Shiokawa et al. (2016) applied market basket analysis framework to visualize transaction data to assess the various human lifestyles. Solnet et al. (2016) also studied the potential of market basket analysis to grow revenue of hotels. To do this, they explored and derived the most attractive services and products where could attract and satisfy hotel guests and encourage them to repeat their purchase. In another research, Coscia et al. (2016) were used market basket analysis approach to explore cultural consumer behaviours. Obviously, the conducted research on the market basket analysis are not limited to those reviewed papers and for more information interested reader may refer to Holý et al. (2017), Olson (2016), Aguinis et al. (2013), Kaur and Kang (2016). However, reviewing the related research reveals that the main goal of the market basket analysis and its application is optimization. For more information about the application of optimization models in data mining and application of evolutionary algorithms in association rule mining, please refer to Tomar and Manjhvar (2015) and Shrivastava and Rajput (2015).

Although there may be a considerable number of research on the association rules mining techniques and optimization methods in a separate research, however, to the best of our knowledge, mathematical optimization methods alongside association rule mining as well as applying meta-heuristic methods has not been properly incorporated to formulate the market basket analysis problem. In this paper, we present 
a non-linear zero-one optimization model for mining association rules and placing products on shelves. It is worth to note that, the proposed mathematical model and applying proper meta-heuristic is not addressed in the previous research and we believe that the proposed model provide a comprehensive framework to a more realistic formulation of the real world problems.

The organization of this paper is as follows: in the next section, problem description and formulation is presented. In the third section, a Genetic Algorithm is used to solve proposed model. An illustrative example is provided to clarify the proposed model in the fourth section. Finally, conclusions are remarked.

\section{Problem description and formulation}

Consider market data logs that include the items purchased by the customers. The manager of a supermarket wants to maximize the interestingness of the product placement on shelves. That is, interestingness value related to mined association rules and the location of shelves. The rationale of interestingness maximization with location considerations is based on this fact that, association rules mining helps to maximize cross-selling effect however it is clear that the location of shelves has the undeniable impact on the selling rate. For example, the products that are placed into near the entrance or exit doors have more chance to be purchased. So, it can be said that the preference function of the supermarket manager depends on the following parameters: selling the benefit, support, and confidence of each pair of products and the selling possibility of each shelf for each product. These parameters are integrated into the following preference function:

Preference function:

$$
\sum_{i=1}^{m-1}\left[\sum_{l=i+1}^{m}\left[C_{i l}+C_{l i} \sum_{k=1}^{P}\left[b_{i} v_{i k}+b_{l} v_{l k} x_{i k} x_{l k}\right]\right]\right]
$$

where $m$ is the number of products, $P$ is the number of shelves, $C_{i l}$ is the confidence of the rule (product $i \rightarrow$ product $l$ ), $b_{i}$ also is selling benefit of the $i$ h product, $v_{i k}$ is selling possibility degree of the product $i$ when placed into the $k$ th shelf and $x_{i k}$ is the binary decision variable that takes 1 when product $i$ is allocated to shelf $k$, otherwise $x_{i k}$ will be 0 .

As it is expected, there are some restrictions that limit the preference function value. At first, the capacity limitation of each shelf must be considered as the following constraint.

$$
\sum_{i=1}^{m} x_{i k} \leq U_{k} \quad ; k=1,2, \ldots, P
$$

where $U_{k}$ is the capacity of the $k$ th shelf. The second constraint is the association constraint: Support of the rule (product $i \rightarrow$ product $l$ ) must greater than the minimum threshold determined by the decision maker.

$$
x_{i k} x_{l k}\left(S_{i l}-S_{\text {min }}\right) \geq 0 ; \forall i, l \in\{1,2, \ldots, m\}
$$


where $S_{i l}$ is the support of rule (product $i \rightarrow$ product $l$ ) and $S_{\min }$ is the minimum support. The third constraint says each product can be allocated to just one shelf according to the following equation.

$$
\sum_{k=1}^{P} x_{i k}=1 ; i=1,2, \ldots, m
$$

According to this fact that the objective and constraints are non-linear functions in which decision variables are binary; we deal with a rough feasible space that increases the probability of trapping in the local optimum. Moreover, it could be proved that the developed model belongs to a class of computationally hard problems which is called Np-hard problems. So in the next section, a GAs based solution approach is developed to solve the proposed mathematical model.

\section{GAs based solution approach}

Genetic algorithm belongs to a category of meta-heuristics methods known as stochastic search ones that use a randomized choice of operators in its search strategy. In this section, we implement GAs to obtain a solution for presented model in the previous section. The general mechanism of the GAs is depicted in Figure 1.

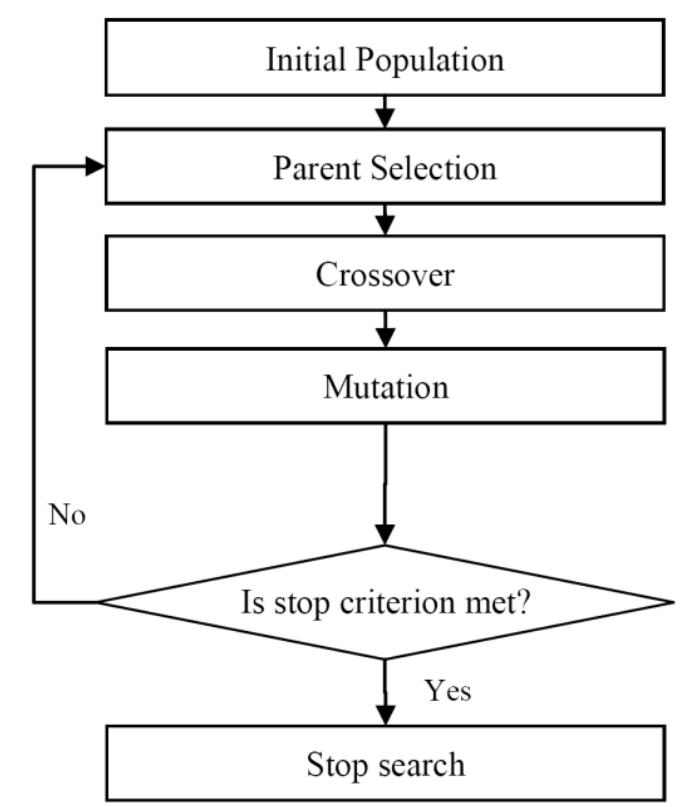

Figure 1. Genetic Algorithm mechanism

Source: Authors' own processing.

It has been proved that the characteristics of the GA such as crossover, mutation and penalty function as well as the selection mechanisms has a major impact on the quality of the provided solutions. For implementing the GA, we need to determine the following essential concepts: chromosome representation, crossover and mutation and selection strategies. 


\section{Chromosome representation}

A chromosome shows the structure of the solution. The considered chromosome for each solution is depicted in Figure 2. in which the value of each gene is binary that takes 1 when product $i$ is allocated to shelf $k$, otherwise 0 .

\begin{tabular}{|l|l|l|l|l|l|l|l|l|}
\hline$x_{11}$ & $x_{12}$ & $\ldots$ & $x_{1 P}$ & $\ldots$ & $x_{m 1}$ & $x_{m 2}$ & $\ldots$ & $x_{m P}$ \\
\hline
\end{tabular}

Figure 2. Chromosome structure

\section{Crossover and mutation}

Source: Authors' own processing.

In the GA, crossover and mutation operators are used to discover the unknown regions of the feasible space. Primarily, the crossover operator is considered as an exploitation mechanism while mutation is used for exploration of the feasible space. As shown in Figure 3, the position based crossover is used. A set of positions from the first parent is selected at random. These values are copied on the same situations of offspring and remained ones are fulfilled by the same genes of the second parent. Another offspring is generated in the same manner.

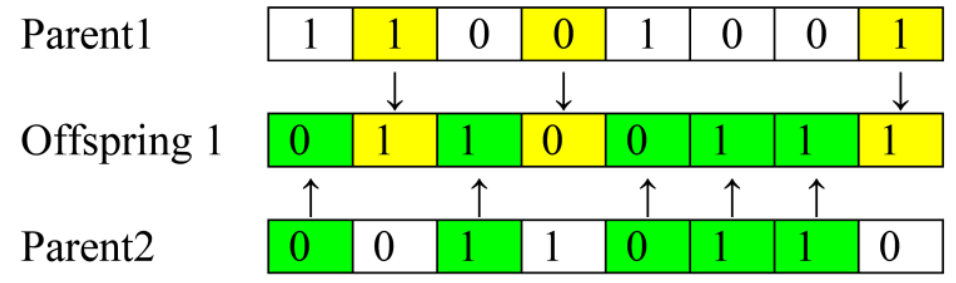

Figure 3. Crossover operator

Source: Authors' own processing.

Mutation operator is organized in a way that one position is selected at random and its value is flip-flopped. Its mechanism is depicted in Figure 4.

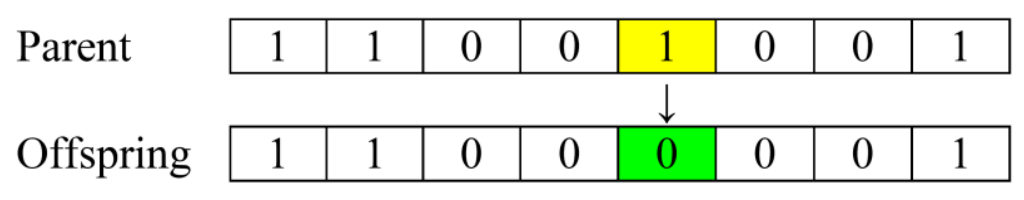

Figure 4. Mutation operator

Source: Authors' own processing.

\section{Selection strategies}

Here we use two selection strategies, roulette wheel for selecting parents to generate offspring and elitism to select next generation from the offspring and parents as survivor selection mechanism. Interested readers are referred to Sivanandam and Deepa (2008) for more information about roulette wheel and elitism mechanisms.

\section{Feasibility checking and fitness evaluation}

In the optimization problems with discrete feasible space, generating feasible solutions is a major concern. There are two approaches to deal with this problem. The first is searching the feasible space via generating feasible solutions that are a time-consuming 
way and also may be not lead to effective exploitation and exploration. Another approach could be a use of the penalty function. In this way, all produced offspring either feasible or infeasible are accepted and the penalty value is assigned to infeasible solutions based on their infeasibility degree. The infeasibility degree is computed based on the proportion of the violated constraints. So the fitness value of the each offspring is included the objective function and the penalty values.

In the next section, an illustrative numerical example is presented to clarify the developed model and proposed solution approach.

\section{Numerical example}

In this section, an example of market basket data is simulated to describe the proposed model and GAs based solution approach. To do this, ten goods are considered that must be allocated into three shelves. Based on the shelves position each shelf has a different impact on the selling possibility of the allocated goods. These selling possibilities can be determined by experts. These values are presented in Table 1.

Table 1. The selling possibility of each goods $\left(v_{i k} ; i=1, \ldots, 10, k=1,2,3\right)$

\begin{tabular}{|c|c|c|c|c|c|c|c|c|c|c|}
\hline $\begin{array}{c}\text { Goods } \\
\text { Shelves }\end{array}$ & $\mathbf{1}$ & $\mathbf{2}$ & $\mathbf{3}$ & $\mathbf{4}$ & $\mathbf{5}$ & $\mathbf{6}$ & $\mathbf{7}$ & $\mathbf{8}$ & $\mathbf{9}$ & $\mathbf{1 0}$ \\
\hline 1 & 0.8 & 0.6 & 0.1 & 0.9 & 0.1 & 0.1 & 0.5 & 0.1 & 0.2 & 0.1 \\
\hline 2 & 0.5 & 0.2 & 0.5 & 0.4 & 0.5 & 0.3 & 0.5 & 0.1 & 0.3 & 0.8 \\
\hline 3 & 0.9 & 0.5 & 0.4 & 0.1 & 0.9 & 0.7 & 0.5 & 0.7 & 0.8 & 0.1 \\
\hline
\end{tabular}

Source: Authors' own processing.

Another characteristic that has a major impact on the allocating products is the selling benefit. So, it is logical that for maximizing the expected benefit of the selling, the products with the higher benefits must be allocated to shelves with higher selling possibilities. Table 2 shows the values of the products' benefit.

Table 2. The benefit of each product ( $\$ /$ unit $)\left(b_{i} ; i=1, \ldots, m\right)$

\begin{tabular}{|c|c|c|c|c|c|c|c|c|c|c|}
\hline Goods & $\mathbf{1}$ & $\mathbf{2}$ & $\mathbf{3}$ & $\mathbf{4}$ & $\mathbf{5}$ & $\mathbf{6}$ & $\mathbf{7}$ & $\mathbf{8}$ & $\mathbf{9}$ & $\mathbf{1 0}$ \\
\hline Benefit & 40 & 15 & 70 & 20 & 15 & 25 & 10 & 10 & 22 & 5 \\
\hline
\end{tabular}

Source: Authors' own processing.

For simulated data, the values of confidence and support are obtained as Tables 3 and 4 respectively.

Table 3. The confidence values for simulated data $\left(C_{i l}\right)$

\begin{tabular}{|c|c|c|c|c|c|c|c|c|c|c|}
\hline $\begin{array}{c}\text { Good } \\
\mathbf{S}\end{array}$ & $\mathbf{1}$ & $\mathbf{2}$ & $\mathbf{3}$ & $\mathbf{4}$ & $\mathbf{5}$ & $\mathbf{6}$ & $\mathbf{7}$ & $\mathbf{8}$ & $\mathbf{9}$ & $\mathbf{1 0}$ \\
\hline $\mathbf{1}$ & 1 & 0.67 & 0.42 & 0.44 & 0.36 & 0.28 & 0.28 & 0.47 & 0.31 & 0.33 \\
\hline $\mathbf{2}$ & 0.51 & 1 & 0.38 & 0.38 & 0.38 & 0.28 & 0.23 & 0.47 & 0.3 & 0.3 \\
\hline $\mathbf{3}$ & 0.33 & 0.39 & 1 & 0.48 & 0.43 & 0.26 & 0.3 & 0.46 & 0.37 & 0.3 \\
\hline $\mathbf{4}$ & 0.36 & 0.4 & 0.49 & 1 & 0.36 & 0.31 & 0.29 & 0.53 & 0.4 & 0.27 \\
\hline $\mathbf{5}$ & 0.36 & 0.5 & 0.56 & 0.44 & 1 & 0.22 & 0.25 & 0.44 & 0.39 & 0.36 \\
\hline $\mathbf{6}$ & 0.28 & 0.36 & 0.33 & 0.39 & 0.22 & 1 & 0.56 & 0.53 & 0.28 & 0.28 \\
\hline
\end{tabular}




\begin{tabular}{|c|c|c|c|c|c|c|c|c|c|c|}
\hline $\mathbf{7}$ & 0.32 & 0.35 & 0.45 & 0.42 & 0.29 & 0.65 & 1 & 0.39 & 0.23 & 0.32 \\
\hline $\mathbf{8}$ & 0.35 & 0.45 & 0.43 & 0.49 & 0.33 & 0.39 & 0.24 & 1 & 0.47 & 0.33 \\
\hline $\mathbf{9}$ & 0.35 & 0.45 & 0.55 & 0.58 & 0.45 & 0.32 & 0.23 & 0.74 & 1 & 0.29 \\
\hline $\mathbf{1 0}$ & 0.4 & 0.47 & 0.47 & 0.4 & 0.43 & 0.33 & 0.33 & 0.53 & 0.3 & 1 \\
\hline
\end{tabular}

Source: Authors' own processing.

Table 4. The support values for simulated data $\left(S_{i l}\right)$

\begin{tabular}{|c|c|c|c|c|c|c|c|c|c|c|}
\hline $\begin{array}{c}\text { Good } \\
\mathbf{S}\end{array}$ & $\mathbf{1}$ & $\mathbf{2}$ & $\mathbf{3}$ & $\mathbf{4}$ & $\mathbf{5}$ & $\mathbf{6}$ & $\mathbf{7}$ & $\mathbf{8}$ & $\mathbf{9}$ & $\mathbf{1 0}$ \\
\hline $\mathbf{1}$ & 0.36 & 0.24 & 0.15 & 0.16 & 0.13 & 0.1 & 0.1 & 0.17 & 0.11 & 0.12 \\
\hline $\mathbf{2}$ & 0 & 0.47 & 0.18 & 0.18 & 0.18 & 0.13 & 0.11 & 0.22 & 0.14 & 0.14 \\
\hline $\mathbf{3}$ & 0 & 0 & 0.46 & 0.22 & 0.2 & 0.12 & 0.14 & 0.21 & 0.17 & 0.14 \\
\hline $\mathbf{4}$ & 0 & 0 & 0 & 0.45 & 0.16 & 0.14 & 0.13 & 0.24 & 0.18 & 0.12 \\
\hline $\mathbf{5}$ & 0 & 0 & 0 & 0 & 0.36 & 0.08 & 0.09 & 0.16 & 0.14 & 0.13 \\
\hline $\mathbf{6}$ & 0 & 0 & 0 & 0 & 0 & 0.36 & 0.2 & 0.19 & 0.1 & 0.1 \\
\hline $\mathbf{7}$ & 0 & 0 & 0 & 0 & 0 & 0 & 0.31 & 0.12 & 0.07 & 0.1 \\
\hline $\mathbf{8}$ & 0 & 0 & 0 & 0 & 0 & 0 & 0 & 0.49 & 0.23 & 0.16 \\
\hline $\mathbf{9}$ & 0 & 0 & 0 & 0 & 0 & 0 & 0 & 0 & 0.31 & 0.09 \\
\hline $\mathbf{1 0}$ & 0 & 0 & 0 & 0 & 0 & 0 & 0 & 0 & 0 & 0.3 \\
\hline
\end{tabular}

Source: Authors' own processing.

The capacity of each shelf $\left(U_{k}\right)$ are presented in Table 5.

Table 5. The capacity of shelf $k\left(U_{k}\right)$

\begin{tabular}{|c|c|c|c|}
\hline Shelves & $\mathbf{1}$ & $\mathbf{2}$ & $\mathbf{3}$ \\
\hline Capacity & 2 & 4 & 4 \\
\hline
\end{tabular}

Source: Authors' own processing.

Based on the above information, the market manager decides to maximize his preference function. The problem is formulated using developed mathematical optimization model and described GA with the following parameters is used to solve the optimization model. The rate of mutation and crossover operators are 0.6 and 0.4 respectively. Population size is considered 500 and one percent of the pool including parents and offspring are selected as elites to pass the next generation directly. The GAs is run with maximum 100 generations as a termination criterion. The GA is run with 1000 cycles and the best solution is reported in table 6 as optimal allocation.

Table 6. The optimum solution

\begin{tabular}{|c|c|c|c|c|c|c|c|c|c|c|}
\hline $\begin{array}{c}\text { Goods } \\
\text { Shelves }\end{array}$ & $\mathbf{1}$ & $\mathbf{2}$ & $\mathbf{3}$ & $\mathbf{4}$ & $\mathbf{5}$ & $\mathbf{6}$ & $\mathbf{7}$ & $\mathbf{8}$ & $\mathbf{9}$ & $\mathbf{1 0}$ \\
\hline 1 & 0 & 0 & 0 & 0 & 0 & 1 & 0 & 0 & 0 & 1 \\
\hline 2 & 0 & 0 & 1 & 0 & 1 & 0 & 0 & 1 & 0 & 0 \\
\hline 3 & 1 & 1 & 0 & 1 & 0 & 0 & 1 & 0 & 0 & 0 \\
\hline
\end{tabular}

Source: Authors' own processing. 
For the above solution, the objective value is 306.9. In order to identify that the provided answer by GA is local or global optimum solution, in the first step we conducted sensitivity analysis of the GA parameters. To do this, we applied bigger population size with different initial population generation mechanisms, crossover, mutation, and selection strategies to solve the numerical example. The results revealed that the proposed crossover, mutation and selection strategies provides well-tuned GA in which properly exploit and explore the feasible space. In the second step, since the numerical example had a small scale, we solved the numerical example using GAMS optimization software. The GAMS's result had been show that the derived optimal solution by GA is a global optimum.

\section{Discussion}

Operation research methods have been widely used to model, analyze and optimize the considerable number of raised problems in data mining (Meisel and Mattfeld, 2010). Market basket analyzing as one the most studied and applied area of data mining is of great interest to practitioner and researchers. Reviewing the market basket analysis reveals that the association rule mining and classification, as well as their optimization is the main subject of most reported research. To do this, evolutionary algorithms like simulated annealing, genetic algorithm, particle swarm, ant colony are also widely used to classify, mine and optimize association rules in data sets (Dhaenens and Jourdan, 2016, Sadh and Shukla, 2013). For example, focusing on the application of GA in association rule mining, one can find considerable number of research in which the main subject is limited to optimizing association rules (Sharma and Tivary, 2012; Tiwari and Singh, 2010; Collard and Francisci, 2001; Waiswa and Baryamureeba, 2008; Ghosh et al., 2010). However, extracting support and confidence rules from a set of data may not provide all needed information to effectively address the market basket analysis problem. Since, factors like store layout, shelves layout and placing of goods on shelves also have un-negligible effects on the sales of goods, so considering these factors may help to more realistic analyzing and optimization.

In this paper considering the effects of placing goods on shelves, a new mathematical model was developed. To do this, considering the support and confidence as two important factors of market basket analysis, the products' location was regarded as another parameter which may affect the selling rate. These factors and their effects, in terms of preference function, were formulated as a non-linear zero-one programming model and it was solved using the GA. It is worth to note that, in the most of the reported research, the meta- heuristic algorithms like GA was applied to discover the association rules, while in the present paper, it is assumed that the support and confidence rules of data set were already known. Therefore, instead of focusing on the optimization of association rules, in this paper the GA was used to derive the optimum location of the goods on the shelves in which preference function of the decision maker was maximized.

About the performance and the properties of the developed GA it is worth to note that at one hand, proper setting up the parameters of evolutionary algorithms mainly affects the performances of algorithms. On the other hand, tuning evolutionary algorithms like GA heavily depend on the properties of the mathematical optimization model. Therefore, one can expect that while a well-tuned GA may successfully obtain a globally optimal solution for a given mathematical model, applying this GA for another problem may result in a local and unsatisfactory solution. As a result comparing 
provided GA with other developed evolutionary algorithms on different mathematical models is not the case. However, in order to ensure the performance of the proposed GA, we compared the derived optimal solution by GA with GAMS on small-scale mathematical problems. The provided result revealed that the proposed GA is a welltuned and could successfully use to derive an optimum solution.

In summary, while in the present paper the effect of goods location on the sales has been incorporated and a more realistic solution has been provided, but there are some drawbacks that need to be addressed in future research. In the present paper, association rules assumed to be known in advance while one can use the appropriate method and algorithm to discover the association rules as well. In the present model, it was assumed that the model parameters are crisp and the exact values of the parameters are known. But as one can expect, due to the uncertainty of the environment, such an assumption is not valid and developing uncertain mathematical models may help to derive more a realistic models and in turn solutions. Classifying goods, defining appropriate interestingness criteria are also possible future research directions.

\section{References}

Aguinis, H., Forcum, L.E., and Joo H. (2013), "Using market basket analysis in management research", Journal of Management, Vol. 39, No. 7, pp. 1799-1824.

Badhe, V., and Richharia, P. (2016), "Survey on Association Rule Mining for Finding Frequent Item Pattern", International Journal of Scientific Research in Science, Engineering and Technology, Vol. 2, No. 2, pp. 1349-1355.

Barbu, A. (2013), "Eight contemporary trends in the market research industry", Management \& Marketing, Vol. 8, No. 3, pp. 429-450.

Bradley, P.S., Fayyad, U.M. and Mangasarian, O.L. (1999), "Mathematical programming for data mining: Formulations and challenges", INFORMS Journal on Computing, Vol. 11, pp. 217-238.

Cavique, L. (2007), "A scalable algorithm for the market basket analysis", Journal of Retailing and Consumer Services, Vol. 14, pp. 400-407.

Chen, Y., Tang, K., Shen, R., and Hu, Y. (2005), "Market basket analysis in a multiple store environment", Decision Support Systems, Vol. 40, pp. 339-354.

Collard, M., and Francisci, D. (2001), "Evolutionary Data Mining : an overview of Geneticbased Algorithms", Proceedings 8th IEEE International Conference on Emerging Technologies and Factory Automation, pp. 3-9.

Coscia, C., Fontana, R., and Semeraro, P. (2016), “Market Basket Analysis for studying cultural Consumer Behaviour: AMTP Card-Holders", Statistica Applicata, Vol. 26, No. 2, pp. 73-92.

Dhaenens, C., and Jourdan, L. (2016), "Metaheuristics and Association Rules", in Dhaenes, C. and Jourdan, L. (Eds.), Metaheuristics for Big Data, John Wiley \& Sons, Hoboken, pp. 87-108.

Ghosh, S., Biswas, S., Sarkar, D., and Sarkar, P.P. (2010), "Mining Frequent Item sets using genetic algorithm", International Journal of Artificial Intelligence \& Applications, Vol. 1, No. 4, pp. 133-143.

Hegland, M. (2003), "Algorithms for Association Rules", Lecture Notes in Computer Science, Vol. 2600, pp. 226-234. 
Holý, V., Sokol, O. and Černý, M. (2017), “Clustering Retail Products Based on Customer Behavior", Applied Soft Computing, In Press, Accepted Manuscript, doi. 10.1016/j.asoc.2017.02.004.

Kaur, M., and Kang, S. (2016), "Market Basket Analysis: Identify the Changing Trends of Market Data Using Association Rule Mining", International Conference on Computational Modelling and Security (CMS 2016), Vol. 85, pp. 78-85.

Kotsiantis, S., and Kanellopoulos, D. (2006), "Association Rules Mining: A Recent Overview" GESTS International Transactions on Computer Science and Engineering, Vol. 32, No. 1, pp. 71-82.

Mangasarian, O.L. (1965), "Linear and nonlinear separation of patterns by linear programming", Operations Research, Vol. 13, pp. 444-452.

Meisel, S., and Mattfeld, D. (2010), "Synergies of Operations Research and Data Mining", European Journal of Operational Research, Vol. 206, No. 1, pp. 1-10

Sivanandam, S.N. and Deepa, S.N. (2008), Introduction to genetic algorithms, Springer, Berlin.

Nierop, E., Fok, D. and Franses, P. (2006), "Interaction between shelf layout and marketing effectiveness and its impact on optimizing shelf arrangements", Marketing Science, Vol. 27, No. 6, pp. 1065-1082.

Olafsson, S., Li, X. and Wu, S. (2008), "Operations research and data mining", European Journal of Operational Research, Vol. 187, pp. 1429-1448.

Olson, D.L. (2016), "Market Basket Analysis, Chapter Descriptive Data Mining", Part of the series: Computational Risk Management, pp. 29-41.

Padmanabhan, B. and Tuzhilin, A. (2003), "On the use of optimization for data mining: theoretical interactions and eCRM opportunities", Management Science, Vol. 49, No. 10, pp. 1327-1343.

Rao, M.R. (1971), "Cluster analysis and mathematical programming", Journal of the American Statistical Association, Vol. 66, pp. 622-626.

Russell, R.A. and Urban, T.L. (2010), "The location and allocation of products and product families on retail shelves", Annuals of Operation Research, Vol. 179, No. 1, pp. 131-147.

Sadh, A.S. and Shukla, N. (2013), "Association rules optimization: A survey", International Journal of Advanced Computer Research, Vol. 3, No. 9, pp. 111-115.

Saraf, R. and Patil, S. (2016), "Market-Basket Analysis using Agglomerative Hierarchical approach for clustering a retail items", International Journal of Computer Science and Network Security, Vol. 16, No. 3, pp. 47-56.

Sharma, A. and Tivary, N. (2012), "A survey of association rule mining using genetic algorithm", International Journal of Computer Applications \& Information Technology, Vol. 1, No. 2, pp. 5-11.

Shiokawa, Y., Misawa, T., Date, Y. and Kikuchi, J. (2016), "Application of market basket analysis for the visualization of transaction data based on human lifestyle and spectroscopic measurements", Analytical chemistry, Vol. 88, No. 5, pp. 2714-2719.

Shrivastava, S. and Rajput, V. (2015), "Evolutionary algorithm based association rule mining: A brief survey", International Journal of Innovation in Engineering research and Management, Vol. 2, No.1, pp. 1-7.

Solnet, D., Boztug, Y. and Dolnicar, S. (2016), “An untapped gold mine? Exploring the potential of market basket analysis to grow hotel revenue", International Journal of Hospitality Management, Vol. 56, pp. 119-125. 
Tang, K., Chen, Y. and Hu, H. (2008), "Context-based market basket analysis in a multiple-store environment", Decision Support Systems, Vol. 45, pp. 150-163.

Tiwari, R. and Singh, M.P. (2010), "Correlation-based Attribute Selection using Genetic Algorithm", International Journal of Computer Application, Vol. 4, No. 8, pp. 28-34.

Tomar, N. and Manjhvar, A.K. (2015), "A survey on data mining optimization techniques", International Journal of Science Technology \& Engineering, Vol. 2, No. 6, pp. 130-133.

Vinod, H.D. (1964), "Integer programming and the theory of grouping", Journal of the American Statistical association, Vol. 64, pp. 506-519.

Waiswa, P.P.W. and Baryamureeba, V. (2008), "Extraction of interesting association rules using Genetic Algorithms", International Journal of Computing and ICT Research, Vol. 2, No. 1, pp. 26-33.

Yun, C., Chuang, K. and Chen, M. (2006), "Adherence clustering: an efficient method for mining market-basket clusters”, Information Systems, Vol. 31, pp. 170-186. 